\title{
Collective Motion Pattern Inference via Locally Consistent Latent Dirichlet Allocation
}

\author{
Jialing Zou ${ }^{a}$, Qixiang $\mathrm{Ye}^{a}$, Yanting $\mathrm{Cui}^{a}$, Fang $\mathrm{Wan}^{a}$, Kun $\mathrm{Fu}^{c}$, Jianbin \\ Jiao $^{a, b}$ \\ ${ }^{a}$ University of Chinese Academy of Sciences, Beijing 101408, China. \\ ${ }^{b}$ jiaojb@ucas.ac.cn \\ ${ }^{c}$ Institute of electronics, Chinese Academy of Sciences
}

\begin{abstract}
Extracting motion descriptors in crowd videos is highly challenging due to scene clutter and serious occlusions. In this paper, Locally Consistent Latent Dirichlet Allocation (LC-LDA) model is proposed to learn collective motion patterns using tracklets and bag-of-words as low level features. The LC-LDA model implements a graph Laplacian operator to impose neighboring constraints to tracklets on a local manifold, which enforces the spatial-temporal coherence of tracklets in a high dimensional bag-of-word feature space. With initialization of clustering on a manifold, LC-LDA model improves the unsupervised inference capability and compactness of learned collective motion patterns. Experimental results on three public datasets indicate that LCLDA based motion patterns can improve the trajectory clustering performance effectively.
\end{abstract}

Keywords: Collective Motion, Local Motion Pattern, Topic Model, Trajectory Clustering

\section{Introduction}

Extracting motion patterns or object feature descriptors in computer vision is of great value to activity recognition [1, 2], event detection [3, 4], face detection [5, 6], image classification [7], texture segmentation [8] and scene understanding [9-13]. In crowded video scenes, however, collective motion pattern or feature descriptor extraction remains a challenging task due to 


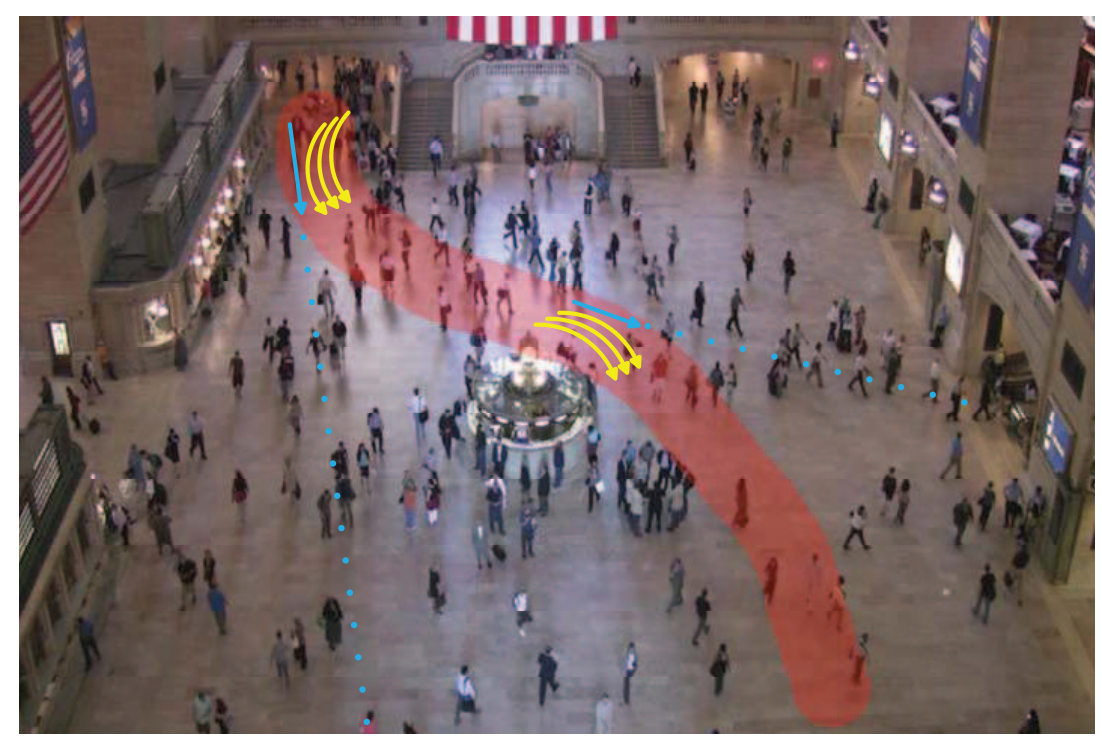

Figure 1: Illustration of tracklets in same space sharing different motion patterns. (Better view in color vision).

frequent occlusions among objects. With existing tracking algorithms $[2,14$ 17], short trajectory segments (tracklets) are usually obtained, from which it is difficult to extract meaningful motion patterns by performing simple trajectory classification [18] or clustering [19]. Zhu et al. [20] propose tracking crowded groups in surveillance videos based on the theory of coherent motions [21] in crowded scenes. They obtain crowded motion patches through a KLT keypoint tracker and then integrate and update the patches into groups. The information in the same level of patches and among hierarchical levels of patches is used to regularize the tracking results.

To "connect" tracklets into meaningful motion patterns, unsupervised inference methods have been widely explored. In [9], a hierarchical unsupervised framework with $k$-means and GMMs is proposed to learn motion patterns from dense optical flow features. In [11], a Tensor Voting algorithm is employed to calculate the local geometry structure of tracklets. In the local geometry structure space, Bayesian inference is later used to perform motion pattern inference. In [22], Chen et al. apply a deep model, Replicated Softmax-based model, to cluster tracklets in crowded scenes. In [4], Wang et al. propose using a mixture of Latent Dirichlet Allocation (LDA) models to learn collective motion patterns. Motion patterns are reflected 
by a hierarchical Dirichlet processes, where collective motions are modeled as distributions over tracklet features, and interactions are modeled as distributions over collective motion patterns. The approach mentioned above explore the advantages of statistical inference; however, few of them consider the spatial-temporal coherence among tracklets. In Figure 1, the red path denotes collective motion patterns. Although it can be seen the red tracklets and the blue trackltes in two clusters respectively are in the same spatialtemporal space, a single tracklet(the blue one in Figure 1) may denote the wrong motion pattern.

To enforce the spatial-temporal coherence of tracklets, scene or object motion priors are used. Zhou et al. [10], Alahi et al. [23] and Zou et al. $[24,25]$ incorporate the scene priors into their frameworks, respectively. Their priors are scene sources and sinks, estimated from the starting and ending points of tracklets. In [26], a Mixture model of Dynamic pedestrianAgents (MDA) is proposed to learn collective behavior patterns of pedestrians in crowded scenes. Each pedestrian in the crowd is driven by a dynamic pedestrian-agent with a motion prior, and the whole crowd is subsequently modeled as a mixture of dynamic pedestrian-agents. Once the model is learned, MDA can be used to simulate crowd motion patterns. Although the usage of scene or object motion priors significantly improves the inference performance, it also reduces the robustness of the model when considering various scenes. In addition, when priors are introduced into the statistical inference framework, the discriminative power of the learned motion patterns may decrease [27], as the sample size increases.

In this paper, we propose a Locally Consistent LDA (LC-LDA) topic model for collective motion pattern inference using tracklets as low level features. The LC-LDA model carries out the cluster initialization on a manifold, updates correlation among topics, and coherence among tracklets with expectation-maximization iteration. We define a symmetrical KL divergence for the distance metric in a probability space. The metric is added to the objective function as a penalty term by mathematical approximation for the convergence of Variational Breaking Algorithm [28]. This term is used to impose neighbor constraints on tracklets and to enforce the spatial-temporal coherence of tracklets that belong to the same motion pattern. In the implementation of LC-LDA algorithm, the penalization term is approximated in the form of graph Laplacian, which is a discrete approximation of Laplace Beltrami operator [29].

Figure 2 exhibits three types of LDA topic models for motion pattern in- 


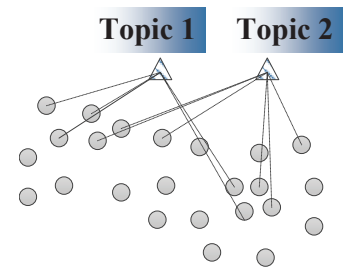

LDA

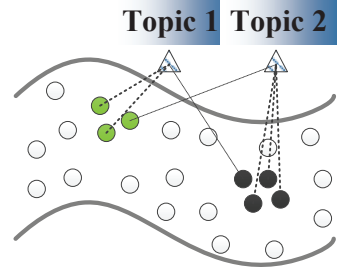

RFT

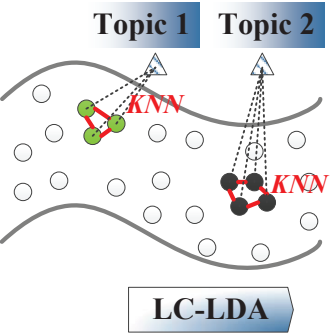

Figure 2: Mapping among tracklets and topics of LDA, RFT, and LC-LDA. Points of different colors denote tracklets of different clusters. (Better view in color vision).
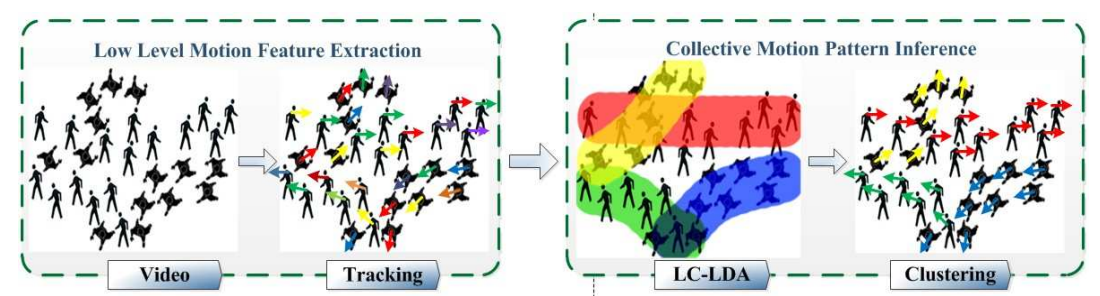

Figure 3: Illustration of the collective motion pattern inference. (Better view in color vision).

ference. In the original LDA model, the input data has no class information, and the LDA model just considers the general likelihood. Therefore, topics (motion patterns) inferred by the LDA model are not discriminative. To obtain discriminative topics, a Random Field Topic (RFT) model [10] is used to introduce data class information by scene priors. However, class information cannot be transferred to topics in the model inference procedure. In [10], it has been proven that the usage of priors could be invalid to the improvement of the discriminative capability of learned topics. In the proposed LC-LDA, a Laplacian manifold embedding is used to capture data class information, which keeps the compactness of initial topics. In the model inference procedure, local consistence constraints are embedded to keep the discriminative capability of learned topics.

Based on LC-LDA topic model, we propose an unsupervised approach for learning collective motion patterns, as well as performing trajectory clustering, as shown in Figure 3. Given a crowded video, tracklets are firstly computed. The tracklets are used to compute linking weights through a spanning tree algorithm [10] and are described with bag-of-word features. Low level motion features are fed to the LC-LDA model to infer motion pat- 
terns, on which we perform tracklet clustering using an entropy clustering algorithm [10].

The contributions of this paper are summarized as follows:

1. We propose a Locally Consistent LDA (LC-LDA) model to improve the inference performance of LDA by introducing locally consistent constraints.

2. We propose using a manifold embedding approach to initialize the LC-LDA model and using an iterative procedure to optimize it, which make it possible to learn collective motion patterns without any scene or object motion prior.

3. Based on the learned collective motion patterns, we develop an effective approach for tracklet clustering in crowded video scenes.

\section{Related Works}

When scene priors are not available, graph and dynamic methods are often employed in motion pattern analysis. In [30], Ali et al. map tracklets to a braid and estimate motion patterns with the graph optimization theory. Mehran et al. [31] propose representing trajectories by streak lines with fluid dynamics. In [32], a social attribute-aware force model is implemented for abnormal event detection. The model can describe the dynamics of interactions among crowds through firstly estimating the scene scale and then creating the interaction model by an online fusion strategy on the basis of social force. In [33], Chong et al. use Hierarchical Dirichlet Processes(HDP) to learn the dominant motion patterns of crowd. They then build histograms on the basis of those patterns to describe time-correlated crowd events. In [34], Wu et al. model crowd directly in both absence and presence of escape events through a dynamic Bayesian framework. They train the model in the events of non-escape behaviors and automatically detect escape events. Those methods ignore the spatial-temporal relations among motion features. They are usually effective for uniform motion patterns. For collective motion patterns, however, they are not the best choice due to randomness and unbalance of motion. In [35], Zhang et al. propose a sparse coding model to learn crowd prototypes and extend a biological model of motion processing. They introduce structural and local coherence constraints in the coding methods. In [36], Yi et al. introduce sparse constraints along spatial-temporal dimensions to shape a 3D stationary time map, which focuses on estimating how long a foreground pixel becomes stationary. These methods effectively use spatial-temporal information to constrain the extraction of motion patterns. 
In [32], Nam et al. group motion flows into crowds through a spatial-temporal grid-based framework. The moving objects' speed and direction are used as motion features which are measured on the grid based graph. However, the sparse coding or grid based graph methods need rules to be pre-defined and can not be smoothly adapted to other scenes. In [24], Zou et al. propose a clustering-specific model by introducing a clustering variable to constrain the topic inference process. For crowd scenes, they initialize the clusteringspecific variable through KNN on the manifold. However, this method ignores the spatial relation updating among tracklets when performing model inference.

Feature descriptors have been specified for motion pattern learning. In [37], Fagette et al. use multi-scale texture-related feature vectors in images to describe the differences between the crowd and the background. In [38], a new measurement called "Coherent Neighbor Invariance" is proposed to analyze the collective motion patterns in crowded scenes. In [21], a new descriptor called "collectiveness" is proposed to indicate the degree of individuals acting as an union in collective motion. Based on the "collectiveness" descriptor, a collective merging algorithm is proposed to detect collective motions from random motions.

In [39], Shi et al. propose an improved LDA to enable integration of object appearance. In [40], Zhao et al. propose a topic random field, which defines a MRF over hidden labels of an image, to enforce the spatial coherence between topic labels for neighbor regions. The two models add variables as location and spatial priors with words to infer topics. Those added variables need to be observed and cannot be updated through computation. In [41], Wang et al. propose a time-continuous model whose topics are associated with a continuous distribution over time-stamps. The topics are influenced by both word co-occurrences and the document time-stamp, which is topical temporal coherence. In [42], Wang et al. propose a spatial LDA model to add the spatial structure as a prior, which helps group visual spatially close words (regions) into the same category. The two models use two variables to constrain the topic inference process spatially or temporally. However, the added variables need to be pre-observed. In [43], a dynamic topic model, termed Markov Clustering Topic Model (MCTM) is employed for the video behavior mining. Built on dynamic Bayesian network models and Bayesian topic models, MCTM can improve the models on sensitivity, robustness and efficiency. In [27], class-specific-simplex LDA (css-LDA), is proposed for image classification and scene understanding. The css-LDA provides greater 
flexibility for modeling discriminative information. By creating a mixture of LDA, one categorical data will be used to train one LDA model in mixture models. Zhu et al. [44] propose a Gibbs max-margin supervised topic model, which is a latent variable classifier to discover hidden semantic structures among data. This model minimizes an expected margin loss, without restrictive assumptions and solving subproblems in SVM. In [45], Wang et al. propose a Spectral-sLDA algorithm through deriving a sufficient condition for the identifiability of sLDA and proving a sample complexity. The algorithm can recover the parameters of supervised sLDA compared to variational approximation [28] or Monte Carlo sampling [46]. In [47], Liao et al. propose a nonparametric Bayesian approach to extend the hierarchical Dirichlet process through incorporating prior variables and latent function's values under Gaussian process. The model can automatically determine the number of topics of HDP and structure from GP. In LDA or LDA extension models, the relations among topics are assumed to be independent. To model the variability among topic proportions, Blei et al. propose the Correlated Topic Model (CTM) [48], where the topic proportions exhibit correlation via a joint Gaussian distribution. CTM has been successfully applied to the problem of tracking in unstructured crowded scenes [49]. In [49], a statistical model is introduced to model both the topical and geometrical structure of the dyadic data that explicitly takes into account the local manifold structure. However, the model cannot be applied to clustering or classification tasks. The task and model in [10] are similar to our work. However, it requires the prior of scenes, which is a nontrivial problem for self-adaptive application across multiple scenes. The solving method in [10] uses Gibbs sampling, which makes it difficult to integrate other terms for the requirement of conjugate symmetry.

\section{Locally Consistent Latent Dirichlet Allocation}

LC-LDA model improves LDA by modifying its graph structure and solving method. In section 3.1, the original LDA model is reviewed. We then present the LC-LDA model and its inference procedure in section 3.2 and 3.3 , respectively. The motion pattern inference and tracklet clustering are detailed in section 3.4 .

\subsection{Latent Dirichlet Allocation}

Four important notations of LDA are the corpus, document, topic and word, which in our case correspond to tracklet set, tracklet, motion pat- 


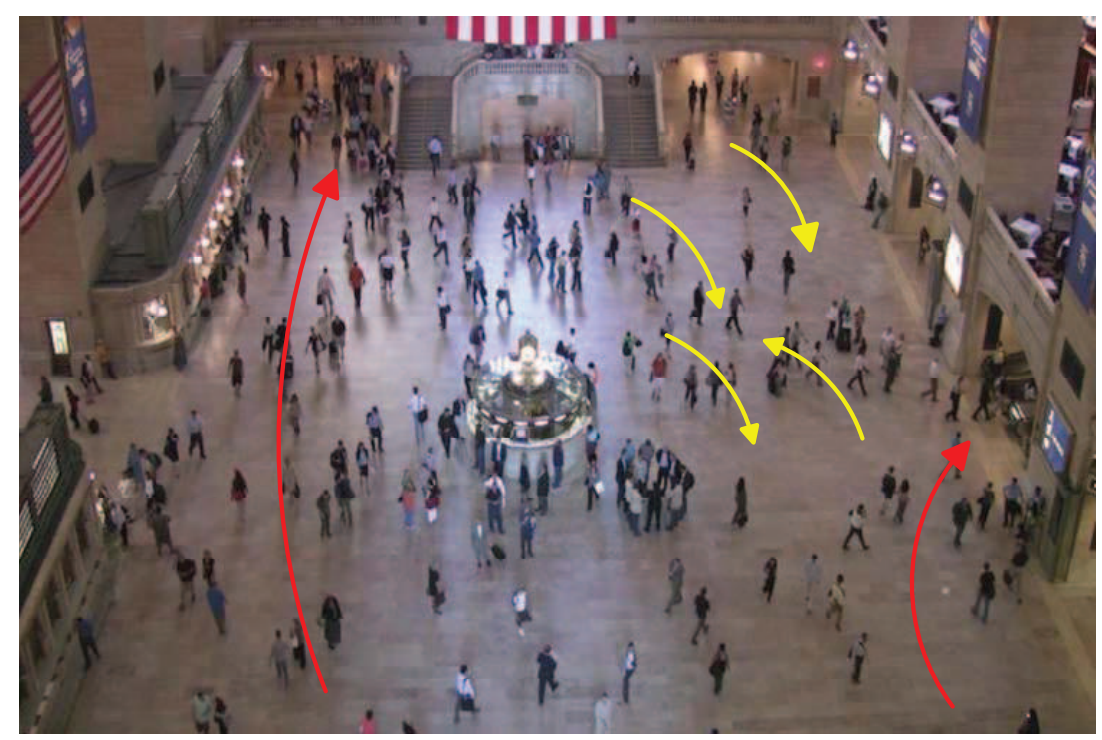

Figure 4: Illustration of different tracklets. (Better view in color vision).

tern and visual word, respectively. Visual words constitute a tracklet, and a tracklet set includes several tracklets. The motion patterns can be extracted within tracklet sets. Figure 4 shows the differences among tracklets. The red ones are longer tracklets which denote that they have more motion information of moving objects than the yellow shorter ones. In this paper, a off-shelf tracking algorithm KLT tracker [50] is used to compute tracklets and details are in section 3.4. Figure 5(a) shows the graphical representation of LDA [51], where $M$ is the number of tracklets. $N_{j}$ is the number of visual words of tracklet $j . Z_{j i}$ is a latent index variable that denotes the motion pattern of the $i t h$ word in the $j t h$ tracklet. It is assumed to follow a parameterized multinomial distribution $Z_{j i} \sim \operatorname{Mult}\left(\theta_{j}\right)$. $\theta$ is assumed to follow a Dirichlet distribution with parameter $\alpha, x$ denotes model inputs, which are the tracklets represented with bag-of-word features (described in the section 3.3). $\beta$ is a hyper-parameter matrix, which describes the collective motion patterns.

\section{2. $L C-L D A$ model}

After a brief review of the conventional LDA model, this section presents the LC-LDA with model formulation, parameter estimation and variable inference.

Based on the notions defined above, Figure 5(b) gives the graphical rep- 


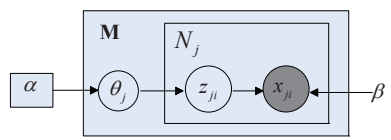

(a)

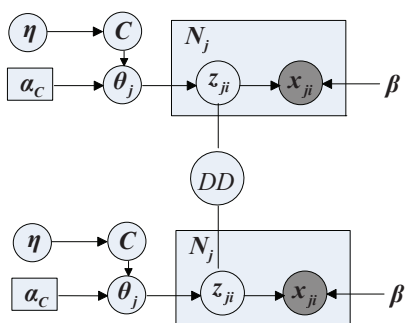

(b)

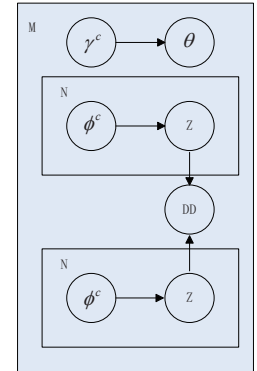

(c)

Figure 5: Graphical representation of (a) LDA, (b) LC-LDA, and (c) approximate distribution of LC-LDA.

resentation of LC-LDA, the joint probability of which is given by

$$
p(x, z, \theta, c \mid \alpha, \beta, \eta)=p(c \mid \eta) \cdot p(\theta \mid \alpha, c) \cdot \prod_{j=1}^{M} p\left(z_{j} \mid \theta_{j}\right) \cdot p\left(x_{j} \mid z_{j}, \beta\right),
$$

where $p(\theta \mid \alpha, c)=\prod_{j=1}^{C} \operatorname{Dir}\left(\theta \mid \alpha_{j}\right)^{\delta(c, j)}$. $\operatorname{Dir}($.$) denotes the Dirichlet distribu-$ tion with parameter $\alpha_{j}$, and the detailed form is $\operatorname{Dir}\left(\theta \mid \alpha_{j}\right)=\frac{\Gamma\left(\sum_{j=1}^{K} \alpha_{j}\right)}{\prod_{j=1}^{K} \Gamma\left(\alpha_{j}\right)}$. $\left(\theta_{j}{ }^{\left(\alpha_{j}-1\right)} \ldots \theta_{K}{ }^{\left(\alpha_{K}-1\right)}\right)$. The terms on the right-hand side of Eq.1 are detailed in the Appendix A. $c$ represents the motion pattern labels of tracklets. Following [52], we set the uniform distribution $P(c)=\frac{1}{C}$, where $C$ is the count of motion patterns. In the inference procedure, $c$ is iteratively updated by maximizing $p(x \mid c, \alpha, \beta)$ and leaving out the estimation of $\eta$. The details of updating variable $c$ are as following. In each iteration of inference, a tracklet can obtain its $\phi$ matrix. The number of rows in the matrix indicates the number of words, and the number of columns, the count of topics. The elements in the matrix indicate the probability of a word under a topic. The matrix $\phi$ is subtracted into a vector by keeping a maximized value in each row, and the vector is subtracted into a single value through choosing a maximized value as the tracklet's topic. The variable $c$, whose dimensionality is equal to the count of topics, is updated with the selected topic index being 1 and the others being 0 .

The motion pattern inference is performed by maximizing the posterior likelihood of $x$ and estimating $\alpha$ and $\beta$, as

$$
p(x \mid \alpha, \beta, c)=\int p(\theta \mid \alpha, c)\left(\prod_{j}^{M} \sum_{z_{j}} p\left(z_{j} \mid \theta_{j}\right) p\left(x_{j} \mid z_{j}, \beta\right)\right) d \theta .
$$


Based on Eq.2, we use the variational breaking algorithm [28] to conduct the variable inference and parameter estimation. According to the approximate distribution of LC-LDA shown in Figure 5(c), we have the log posterior likelihood of $x$, as

$$
\log p(x \mid \alpha, \beta, c)=L\left(\gamma^{c}, \phi^{c} ; \theta^{c}, \beta\right)+K L\left(q\left(\theta, z \mid \gamma^{c}, \phi^{c}\right) \| p\left(\theta, z \mid x, \theta^{c}, \beta\right)\right) .
$$

In the approximated distribution of LC-LDA, $\gamma^{c}$ represents the parameters in the Dirichlet distribution of $\theta^{c} . \phi^{c}$ represents the probability of the motion patterns (topics). In the model inference procedure, we iteratively maximize the term $L($.$) in Eq.3, which results in the minimum of difference between the$ distributions defined in Figure 5(b) and Figure 5(c). The detailed derivation process is provided in Appendix A. We observe that in crowded scenes collective motions always occur in clusters in both time and space. In [53], Cai states that data such as images and text cannot fill up the ambient Euclidean space, because they lie in a group of sub-space. Therefore, we design a metric (symmetrical KL divergence) to measure the distance between tracklets' topics in the probability space. A tracklet's topic is influenced by not only words' co-occurrence, but also the relations among neighbor tracklets' topics, which is denoted in the function $p(z \mid \theta) \propto \exp \left(\sum_{j} \log \theta_{j}+\sum_{j \in \delta(i)} D D\left(z_{j n 1}, z_{i n 2}\right)\right)$. VB algorithm [49] is used to perform parameter estimation and variable inference. According to Figure $5(\mathrm{c}), p(z)$ is directly related to $\phi$. For better presentation, $p(z \mid \theta)$ is presented as $\phi(z)$. The VB algorithm includes three important steps: (1) formulate a derivational likelihood function, (2) estimate parameters in the learning process, and (3) infer variables in the test procedure. The goal is to constrain the topic inference process under the assumption of the coherence of neighbor tracklets. The DD term should be added into the object function. Therefore, a penalization term $R$ is introduced, and $L$ is replaced with $L^{\prime}=L\left(\gamma^{c}, \phi^{c} ; \alpha^{c}, \beta\right)-\lambda \cdot R$, where $\lambda$ is a positive penalization factor. The objective function for model inference is

$$
\begin{gathered}
\max L^{\prime}=\max \left\{L\left(\gamma^{c}, \phi^{c} ; \alpha^{c}, \beta\right)-\lambda \cdot R\right\} \\
R=\frac{1}{2} \sum_{p=1}^{M} \sum_{q=1}^{M}\left(D\left(\phi_{p}^{c}(z) \| \phi_{q}^{c}(z)\right)+D\left(\phi_{q}^{c}(z) \| \phi_{p}^{c}(z)\right)\right) \cdot W_{p, q}, \\
W_{p, q}= \begin{cases}1, & \text { if } d_{p} \in N_{k}\left(d_{q}\right) \text { or } \mathrm{d}_{q} \in N_{k}\left(d_{p}\right), \\
0, & \text { otherwise. }\end{cases}
\end{gathered}
$$


where $D($.$) is the KL divergence measurement between motion patterns'$ probability of the $p^{\text {th }}$ and $q^{\text {th }}$ tracklets under the motion pattern labeled $c$. $W_{p, q}$ is the indicator matrix of the KNN graph, which models the neighboring relationships on a manifold [29]. $N_{k}\left(d_{q}\right)$ denotes the set of KNN of $d_{q}$. M is the number of tracklets. $p$ and $q$ are the indexes of the tracklets. It can be seen that $R$ is symmetrical and can force the neighbor tracklets to share motion patterns.

\subsection{Model Inference}

Model inference is an iterative procedure to maximize $L^{\prime}$ in Eq.4. According to the definition of KL divergence

$$
D\left(\phi_{p}^{c}(z) \| \phi_{q}^{c}(z)\right)=\sum_{z} \phi_{p}^{c}(z) \log \frac{\phi_{p}^{c}(z)}{\phi_{q}^{c}(z)}
$$

we first expand the term $R$ in $L^{\prime}$ as

$$
R=\frac{1}{2} \sum_{p=1}^{M} \sum_{q=1}^{M}\left(\sum_{n}^{N} \sum_{k}^{K} \phi_{p, n, k}^{c} \log \frac{\phi_{p, n, k}^{c}}{\phi_{q, n, k}^{c}}+\sum_{n}^{N} \sum_{k}^{K} \phi_{q, n, k}^{c} \log \frac{\phi_{q, n, k}^{c}}{\phi_{p, n, k}^{c}}\right),
$$

where $K$ is the number of topics, $N$ is the number of visual words in each tracklet, and $M$ is the number of tracklets. If two tracklets are similar, we have $\frac{\phi_{p, n, k}^{c}}{\phi_{q, n, k}^{c}} \approx 1.0$. The reason why the ration approximates to 1 is that we always assume that the neighbor tracklets have coherent topic probability. Therefore, we have $\log \left(\frac{\phi_{p, n, k}^{c}}{\phi_{q, n, k}^{c}}\right) \approx 1-\frac{\phi_{q, n, k}^{c}}{\phi_{p, n, k}^{c}}$, which is the first order expansion of the Taylor series of the log function. Based on this, we simplify Eq.8 as

$$
R=\frac{1}{2} \sum_{p}^{M} \sum_{n}^{N} \sum_{k}^{K} \frac{1}{\phi_{p, n, k}^{c}}\left(\phi_{p, n, k}^{c} \sum_{q}^{M} W_{p, q}-\sum_{q}^{M} \phi_{q, n, k}^{c} W_{p, q}\right) .
$$

The term $\sum_{p}^{M}\left(\phi_{p, n, k}^{c} \sum_{q}^{M} W_{p, q}-\sum_{q}^{M} \phi_{q, n, k}^{c} W_{p, q}\right)$ in Eq.9 can be rewritten as $L \cdot \Phi_{n, k}^{c}$, where $\Phi_{n, k}^{c}=\left[\phi_{1, n, k}^{c} \ldots \phi_{M, n, k}^{c}\right], L=I-W . W$ is a matrix with elements $W_{p, q}$, and $I$ is a diagonal matrix whose elements are the colum$\mathrm{n}$ or row sums of $W\left(W\right.$ is symmetric). $\quad I_{p, p}=\sum_{q} W_{p, q} . \quad L$ refers to the graph Laplacian, which is a discrete approximation to the manifold operator according to [29]. 
We replace the approximated Laplacian form with its log function and compute the derivation of $L$ (detailed in the Appendix A) with respect to $\phi_{p, n, k}^{c}$ and $\log \beta_{n, k}$, based on Eq.4 and Eq.9. The derivations are

$$
\phi_{p, n, k}^{c}=\exp \left(\frac{\log \beta_{n, k} \propto \sum_{p}\left(\log \phi_{p, n, k}^{c}+\log n_{p},\right),}{\left.1+\lambda \cdot \sum_{q=1}^{c}\right)-\Psi\left(\sum_{k=1}^{K} \gamma_{k}^{c}\right)+\log \beta_{k, v}+\lambda \sum_{q=1}^{M} \sum_{k}^{K} \log \phi_{q, n, k}^{c} W_{p, q}-1+\rho_{p, n}}\right),
$$

which correspond to motion patterns and their probabilities, respectively. In Eq.11, $\rho_{i n}$ is the Lagrange multiplier that guarantees $\sum_{k}^{K} \phi_{p, n, k}^{c}=1.0$.

According to Eq.4 and Eq.8, if $\phi_{p, n, k}^{c}$ and $\phi_{q, n, k}^{c}$ of two neighbor tracklets have significant difference then the objective function $L^{\prime}$ has a significant loss. To reduce the loss and maximize $L^{\prime}$ in model inference, label $c$ in $\phi_{p, n, k}^{c}$ is updated iteratively so that neighbor tracklets have similar motion pattern labels $\phi_{p, n, k}^{c}$. According to Eq.10, the results of the motion pattern inference are also related to $\phi_{p, n, k}^{c}$. In other words, if $\phi_{p, n, k}^{c}$ has local consistency, so do the motion patterns.

In [27], it is found that with increase of visual word count $n_{p}$, the discriminative capability of the learned motion patterns decreases. This is consistent with Eq.10. For the state-of-the-art RFT model in [10], the tracklets clustering primarily depends on the motion patterns $\left(\beta_{n, k}\right.$ in Eq.9). However, for the existing term $\log n_{p}$, priors cannot completely regularize the motion patterns $\beta_{n, k}$, according to Eq.10. In addition, the RFT model relies on the sparsity of motion patterns $\beta_{n, k}$ to improve the discriminative capability. We rewrite the motion patterns' probability in the RFT model as

$$
\phi_{k i}^{c} \propto \beta \cdot \exp \left[\Psi\left(\gamma_{k}^{c}\right)-\Psi\left(\sum_{k=1}^{K} \gamma_{k}^{c}\right)\right] \omega_{k i} \cdot \psi_{k i},
$$

where $\omega_{k i}$ and $\psi_{k i}$ are sparse vectors, denoting the sources' and sinks' priors, respectively. Sparse $\omega_{k i}$ and $\psi_{k i}$ bring sparsity to $\phi_{k i}^{c}$ and $\beta_{n k}$. So far, our LCLDA model captures the local consistency reflected by $\phi_{p, n, k}^{c}$, which indicates neighbor relations among tracklets.

\subsection{Motion Pattern Inference and Tracklet Clustering}

Based on the proposed LC-LDA topic model, we propose a framework for motion pattern inference and tracklet clustering, as shown in Figure 6. 


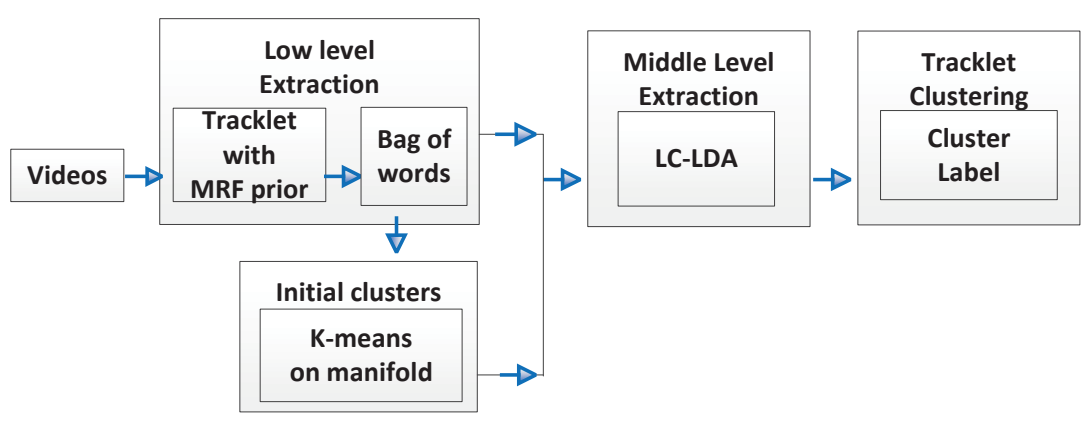

Figure 6: Flowchart of the proposed tracklet clustering approach.

Given a video, we first use a KLT tracker [50] to obtain tracklets. An MRF prior-based spanning tree algorithm is used to link tracklets into tracklet trees [10], on which visual words are extracted as low level features. We construct a codebook for each video scene. The scene image is divided into cells of $10 * 10$ pixels, and the velocity of each tracklet point is quantized into 5 bins, as $v \in\{1,2,3,4,5\}$. Given a scene video resolution of $W * H$, the size of the codebook is set to $\frac{W}{10} * \frac{H}{10} * 5$. With the codebook, we compute a word for each tracklet point, i.e. word $=v *\left(\frac{H}{10} * \frac{W}{10}\right)+\frac{x}{10} * \frac{H}{10}+\frac{y}{10}$, where $(x, y)$ is the coordinate, and $v$ is the velocity bin. With the words for points, each tracklet is represented with bag-of-word features. To initialize the motion pattern labels, we use the Laplacian Eigenmap method [29] to construct a tracklet manifold embedding with $k$ dimensions, which is set to 4 in our scenes. In the manifold embedding space, $k$-means algorithm is adopted to initialize the motion pattern labels. Using the initial labels and low level features as inputs, collective motion patterns can be inferred by the proposed LC-LDA model. In the procedure, the topic probabilities of tracklets in the topic space are computed. Given $K$ topics, each tracklet has $K$ corresponding probabilities, and the topic label of the largest probability is assigned to the tracklet. After this step, each tracklet has a topic label. The topic labels are fed to the information entropy clustering algorithm [10], which computes the potential information entropy of the tracklets and then determines their cluster labels.

\section{Experiments}

Experiments are conducted on three public surveillance video datasets, which are collected from the crowded New York Grand Central Station [10], a 
campus [54] and a cross road [55]. For simplicity, we use "Station", "Campus" and "Cross Road" to denote these three datasets, respectively. The general information of the datasets is shown in Table 1.

Table 1: Datasets

\begin{tabular}{|c|c|c|c|c|}
\hline Dataset & Resolution & Time-Length & Codebook size & Trajectories \\
\hline Station[10] & $720 * 480$ & $1800 \mathrm{~s}$ & $72 \times 48 \times 5$ & 47866 \\
\hline Campus[54] & $360 * 288$ & $216 \mathrm{~s}$ & $36 \times 29 \times 5$ & 32455 \\
\hline Cross Road[55] & $720 * 576$ & $373 \mathrm{~s}$ & $72 \times 58 \times 5$ & 51136 \\
\hline
\end{tabular}

\subsection{Collective motion pattern inference}

In the collective motion pattern inference, we qualitatively evaluate the LC-LDA model and compare it with the state-of-the-art RFT model [10]. Figure 7 shows the learned motion patterns in Station. Our proposed LCLDA model's results are competitive to those of the RFT model [10], even when the motions are complex and overlapped.

Figure 8(a) and (b) show the learned motion patterns in Cross Road. Figure 8(c) and (d) show the learned motion patterns in Campus. In Figure 8 (a) and Figure 8(b), each object (car) follows the traffic routines, therefore most of the motions are regular and not overlapped. When learning the motion patterns in this scene, the locally consistent constraints might not completely regularize the motion patterns. Consequently, the motion patterns elucidated by the LC-LDA model are not very discriminative. In Figure 8(c) and Figure 8(d), most of the tracklets are centered in the middle of the scene, and the neighbor relations are neither complex nor overlapped. Both RFT and LC-LDA models learn clear motion patterns.

Table 2: Tracklet Information

\begin{tabular}{|c|c|c|c|}
\hline Dataset & Completeness & Correctness & Average length \\
\hline Station[10] & 1507 & 2000 & 133 \\
\hline Campus[54] & 500 & 500 & 159 \\
\hline Cross Road[55] & 1000 & 1000 & 185 \\
\hline
\end{tabular}



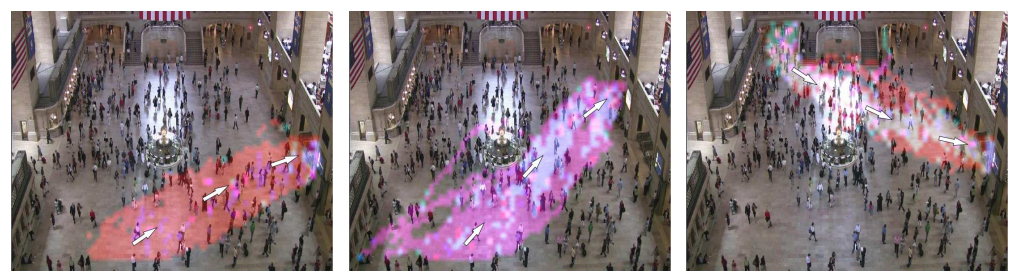

(a)
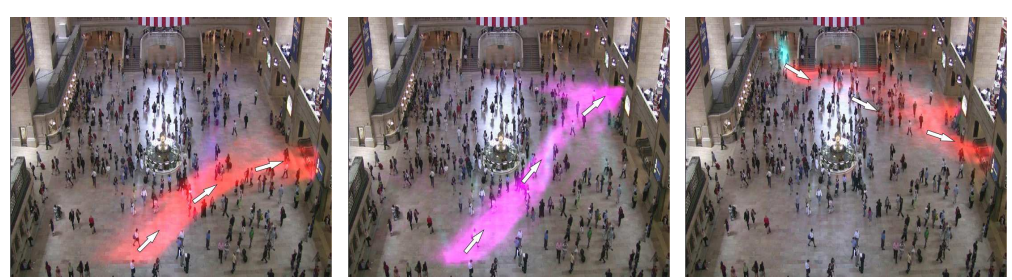

(b)
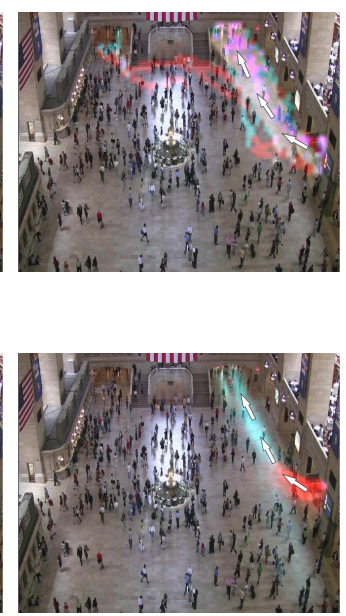

a

Figure 7: Visualization of the learned collective motion patterns in the Station scene. (a) and (b) are learned by LC-LDA and RFT, respectively. (Better view in color vision)

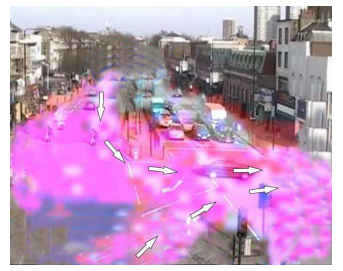

(a)

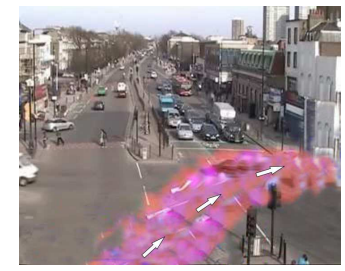

(b)

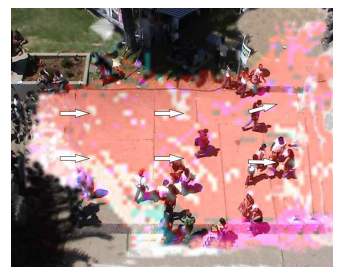

(c)

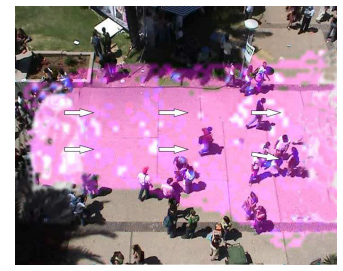

(d)

Figure 8: Visualization of the learned collective motion patterns. (a) and (b) are learned by LC-LDA and RFT in the Cross Road, respectively. (c) and (d) are learned by LC-LDA and RFT in the Campus, respectively. (Better view in color vision)

\subsection{Tracklet clustering}

For tracklet clustering, correctness and completeness [10] are used to measure the performance, and completeness measures how accurately the tracklets from the same clusters are clustered together. Correctness measures how accurately the tracklets from different clusters are divided. For example, if all tracklets are clustered into one single cluster, the completeness is $100 \%$ and the correctness is 0 , and vice versa. We manually label tracklets as ground truth, which is described in Table 2.

Six approaches including 'LC-LDA', 'CLDA[52]', 'MLDA[4]', 'SLDA[42]', 
'RFT[10]', 'CTM[48]' are used for quantitative experiments and comparison. CLDA, SLDA, and MLDA are used for supervised classification. For comparison with other methods in unsupervised tracklet clustering, the variable $c$ in those models is unobserved and initialized with $k$-means. In the completeness accuracy test (Table 3), LC-LDA outperforms the other five approaches, under the similar correctness accuracy in the Table 4 . In Station, the object's behaviors happen simultaneously in disorder. LC-LDA has the initialization with tracklets on Manifold embedding. And the local structure between tracklets on the neighbor graph can be discovered by the locally consistent constraints. In Table 3, the CTM based approach reports lower completeness accuracy than our approach, because it does not fully consider the spatial-temporal consistency of motion patterns (topics). The RFT based approach also reports lower completeness accuracy because it does not update the neighbor tracklets topic information, which is crucial for topic model inference. 'CLDA' and 'SLDA' obtain similar completeness accuracy for the reason of initialization on Manifold Embedding, although the unobserved variable $c$ in 'CLDA' and 'SLDA' have different effects on regularizing parameter $\theta$ 's sampling distribution. The approach 'MLDA' obtains lower completeness accuracy for the reason that the model prefers scenes with dominant paths and have no initialization with tracklet on Manifold Embedding. In Cross Road, though all the behaviors happen in different time, they overlap in the central space of the scene. It can be seen that the approach 'LC-LDA' still outperforms other approaches (Table 7 and Table 8). In Table 5 and Table 6, the approaches 'LC-LDA', 'RFT', 'CLDA', 'MLDA', and 'SLDA' obtain comparable completeness and correctness accuracies, respectively. The reason 'LC-LDA' doesn't advance other approaches is that there is a dominant path in the scene, therefore the structure is simple among tracklets, which can be learned by other approaches.

In Figure 9, we visualize some tracklet clusters by the six approaches. The proposed LC-LDA can calculate clear tracklet clusters. In contrast, the tracklet clusters learned by other approaches are not as good as those by LC-LDA, particularly in the crowded Station. 
Table 3: Accuracy of Completeness in Station

\begin{tabular}{|c|c|c|c|c|c|c|}
\hline Topic & LC-LDA & CTM & RFT & CLDA & MLDA & SLDA \\
\hline 2 & 0.998 & 0.935 & 0.935 & 0.871 & 0.875 & 0.871 \\
\hline 5 & 0.929 & 0.882 & 0.818 & 0.831 & 0.848 & 0.841 \\
\hline 8 & 0.892 & 0.823 & 0.804 & 0.803 & 0.814 & 0.843 \\
\hline 11 & 0.868 & 0.796 & 0.774 & 0.796 & 0.807 & 0.816 \\
\hline 14 & 0.826 & 0.796 & 0.762 & 0.776 & 0.796 & 0.796 \\
\hline 17 & 0.796 & 0.752 & 0.748 & 0.726 & 0.765 & 0.756 \\
\hline 20 & 0.757 & 0.686 & 0.718 & 0.723 & 0.766 & 0.753 \\
\hline 23 & 0.737 & 0.655 & 0.726 & 0.701 & 0.745 & 0.721 \\
\hline 26 & 0.738 & 0.657 & 0.708 & 0.641 & 0.687 & 0.681 \\
\hline 29 & 0.742 & 0.667 & 0.631 & 0.689 & 0.767 & 0.672 \\
\hline 32 & 0.722 & 0.637 & 0.614 & 0.625 & 0.817 & 0.625 \\
\hline
\end{tabular}


Table 4: Accuracy of Correctness in Station

\begin{tabular}{|c|c|c|c|c|c|c|}
\hline Topic & LC-LDA & CTM & RFT & CLDA & MLDA & SLDA \\
\hline 2 & 0.527 & 0.464 & 0.513 & 0.402 & 0.564 & 0.381 \\
\hline 5 & 0.822 & 0.795 & 0.804 & 0.777 & 0.825 & 0.697 \\
\hline 8 & 0.893 & 0.875 & 0.898 & 0.858 & 0.855 & 0.778 \\
\hline 11 & 0.939 & 0.929 & 0.915 & 0.908 & 0.879 & 0.848 \\
\hline 14 & 0.948 & 0.938 & 0.914 & 0.944 & 0.927 & 0.894 \\
\hline 17 & 0.949 & 0.958 & 0.927 & 0.941 & 0.948 & 0.921 \\
\hline 20 & 0.961 & 0.963 & 0.940 & 0.947 & 0.953 & 0.927 \\
\hline 23 & 0.974 & 0.965 & 0.945 & 0.951 & 0.955 & 0.941 \\
\hline 26 & 0.971 & 0.972 & 0.949 & 0.947 & 0.969 & 0.947 \\
\hline 29 & 0.971 & 0.971 & 0.963 & 0.961 & 0.969 & 0.951 \\
\hline 32 & 0.977 & 0.971 & 0.960 & 0.941 & 0.969 & 0.941 \\
\hline
\end{tabular}

Table 5: Accuracy of Completeness in Campus

\begin{tabular}{|c|c|c|c|c|c|c|}
\hline Topic & LC-LDA & CTM & RFT & CLDA & MLDA & SLDA \\
\hline 2 & 0.963 & 0.838 & 0.847 & 0.962 & 0.862 & 0.962 \\
\hline 3 & 0.901 & 0.717 & 0.798 & 0.894 & 0.694 & 0.894 \\
\hline 5 & 0.782 & 0.652 & 0.607 & 0.652 & 0.632 & 0.652 \\
\hline 7 & 0.696 & 0.559 & 0.581 & 0.581 & 0.553 & 0.581 \\
\hline 8 & 0.637 & 0.549 & 0.594 & 0.542 & 0.532 & 0.543 \\
\hline
\end{tabular}


Table 6: Accuracy of Correctness in Campus

\begin{tabular}{|c|c|c|c|c|c|c|}
\hline Topic & LC-LDA & CTM & RFT & CLDA & MLDA & SLDA \\
\hline 2 & 0.631 & 0.441 & 0.467 & 0.482 & 0.442 & 0.422 \\
\hline 3 & 0.724 & 0.699 & 0.645 & 0.539 & 0.679 & 0.499 \\
\hline 5 & 0.848 & 0.802 & 0.835 & 0.711 & 0.801 & 0.671 \\
\hline 7 & 0.898 & 0.841 & 0.876 & 0.794 & 0.834 & 0.754 \\
\hline 8 & 0.934 & 0.863 & 0.911 & 0.787 & 0.857 & 0.747 \\
\hline
\end{tabular}

Table 7: Accuracy of Completeness in Cross Road

\begin{tabular}{|c|c|c|c|c|c|c|}
\hline Topic & LC-LDA & CTM & RFT & CLDA & MLDA & SLDA \\
\hline 2 & 0.993 & 0.838 & 0.985 & 0.975 & 0.848 & 0.955 \\
\hline 3 & 0.949 & 0.668 & 0.949 & 0.934 & 0.665 & 0.924 \\
\hline 5 & 0.861 & 0.673 & 0.838 & 0.723 & 0.663 & 0.713 \\
\hline 7 & 0.832 & 0.572 & 0.716 & 0.692 & 0.602 & 0.682 \\
\hline 8 & 0.833 & 0.621 & 0.722 & 0.681 & 0.631 & 0.671 \\
\hline
\end{tabular}

Table 8: Accuracy of Correctness in Cross Road

\begin{tabular}{|c|c|c|c|c|c|c|}
\hline Topic & LC-LDA & CTM & RFT & CLDA & MLDA & SLDA \\
\hline 2 & 0.814 & 0.648 & 0.773 & 0.814 & 0.698 & 0.824 \\
\hline 3 & 0.871 & 0.781 & 0.854 & 0.851 & 0.811 & 0.849 \\
\hline 5 & 0.929 & 0.878 & 0.883 & 0.909 & 0.868 & 0.907 \\
\hline 7 & 0.971 & 0.889 & 0.949 & 0.931 & 0.879 & 0.969 \\
\hline 8 & 0.981 & 0.913 & 0.955 & 0.951 & 0.893 & 0.982 \\
\hline
\end{tabular}




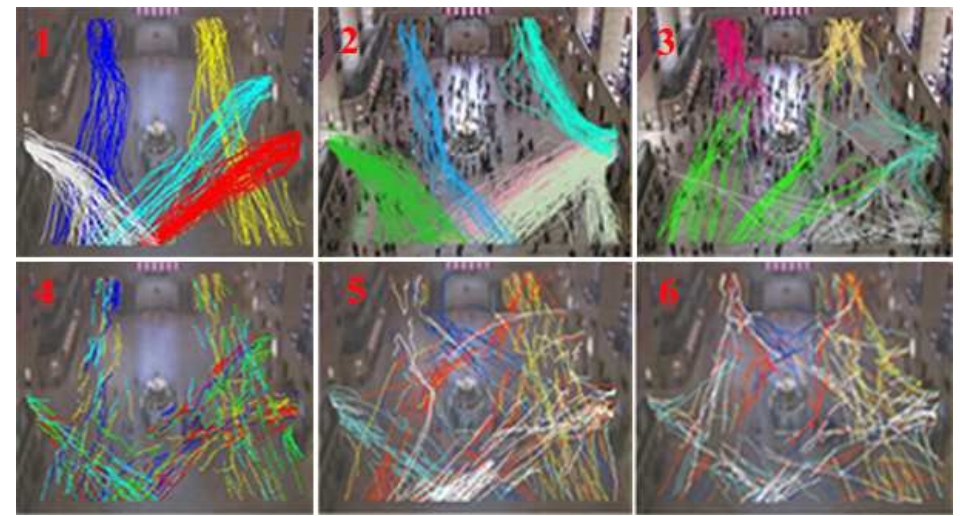

(a)

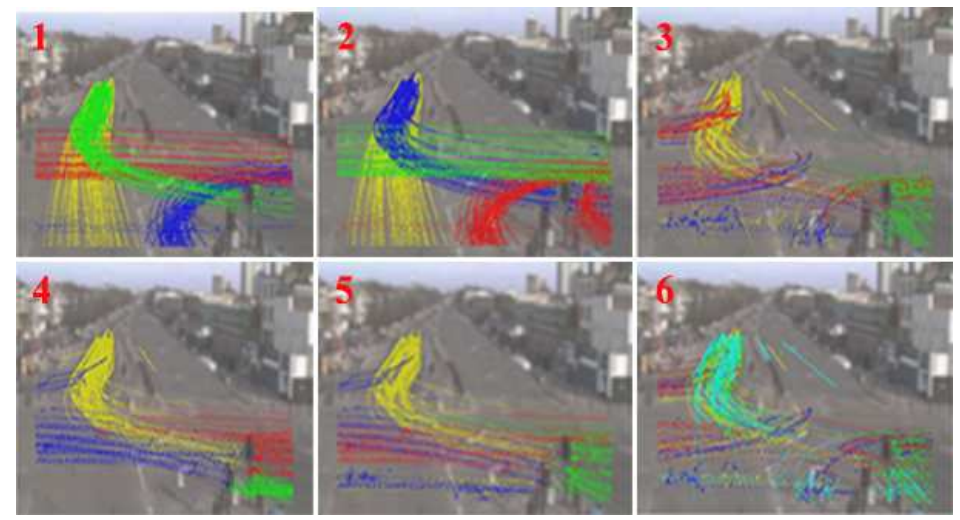

(b)

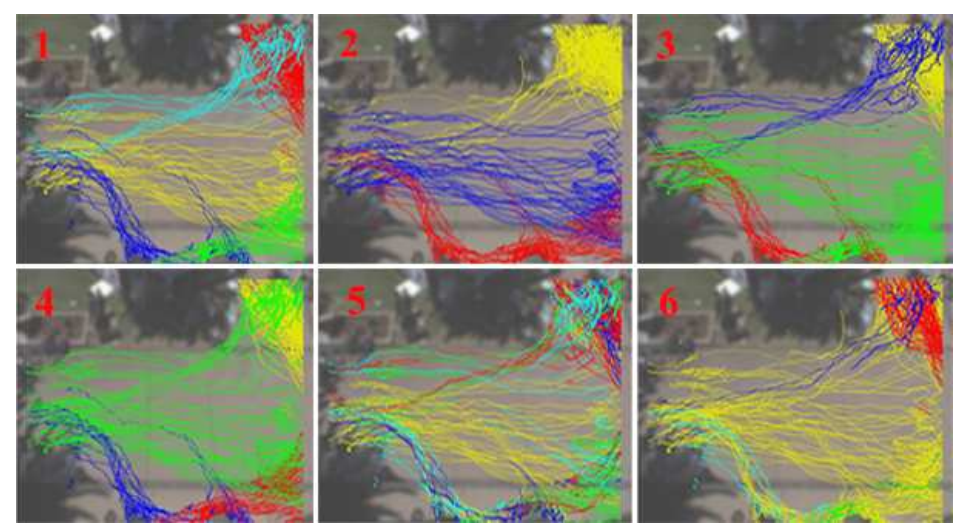

(c)

Figure 9: Tracklet clusters calculated with six approaches. 1. "LC-LDA", 2. "RFT", 3. "CLDA", 4. "CTM", 5."SLDA", 6. "MLDA". (Better view in color vision). 


\section{Conclusion}

We have proposed a Locally Consistent Latent Dirichlet Allocation model for collective motion pattern inference. A symmetrical KL divergence measurement, which is an approximation to a graph Laplacian on a manifold, is introduced into the model inference procedure to enforce spatial-temporal local consistency of tracklets. We demonstrate that the LC-LDA model can infer more discriminative and compact motion patterns. Based on the LC-LDA model, we propose an unsupervised framework to conduct tracklet clustering in crowded video scenes. Experiments and comparison with three datasets show that our approach based on LC-LDA improves the state of the art [10]. In addition, our model and approach do not incorporate scene or object motion priors. In some extremely simple scenes, the LC-LDA cannot significantly advance the classic methods because there is no mixed neighbor relations to be discovered by the local constraint. In the future work, we will consider to handle scenes with dominant paths and improve computation speed in model's learning procedure.

\section{Acknowledgment}

This work is supported in Part by National Basic Research Program of China (973 Program) with No. 2011CB706900 and National Science Foundation of China with Nos. 61271433 and 61202323 


\section{Reference}

[1] F. Jiang, S. Zhang, S. Wu, Y. Gao, D. Zhao, Multi-layered gesture recognition with kinect, Journal of Machine Learning Research 27 (2015) $227-254$.

[2] S. Zhang, H. Yao, X. Sun, K. Wang, J. Zhang, X. Lu, Y. Zhang, Action recognition based on overcomplete independent components analysis, Information Sciences 281 (2014) 635-647.

[3] W. Hu, X. Xiao, Z. Fu, D. Xie, T. Tan, S. Maybank, A system for learning statistical motion patterns, Pattern Analysis and Machine Intelligence (PAMI), IEEE Transactions on 28 (9) (2006) 1450-1464.

[4] X. Wang, X. Ma, W. E. L. Grimson, Unsupervised activity perception in crowded and complicated scenes using hierarchical bayesian models, Pattern Analysis and Machine Intelligence (PAMI), IEEE Transactions on 31 (3) (2009) 539-555.

[5] Z. Lei, M. Pietikainen, S. Li, Learning discriminant face descriptor, Pattern Analysis and Machine Intelligence, IEEE Transactions on 36 (2) (2014) 289-302.

[6] S. Xie, S. Shan, X. Chen, J. Chen, Fusing local patterns of gabor magnitude and phase for face recognition, Image Processing, IEEE Transactions on 19 (5) (2010) 1349-1361.

[7] J. Chen, G. Zhao, M. Salo, E. Rahtu, M. Pietikainen, Automatic dynamic texture segmentation using local descriptors and optical flow, Image Processing, IEEE Transactions on 22 (1) (2013) 326-339.

[8] J. Chen, S. Shan, C. He, G. Zhao, M. Pietikainen, X. Chen, W. Gao, Wld: A robust local image descriptor, Pattern Analysis and Machine Intelligence, IEEE Transactions on 32 (9) (2010) 1705-1720.

[9] I. Saleemi, L. Hartung, M. Shah, Scene understanding by statistical modeling of motion patterns, in: Computer Vision and Pattern Recognition (CVPR), IEEE Conference on, 2010, pp. 2069-2076.

[10] B. Zhou, X. Wang, X. Tang, Random field topic model for semantic region analysis in crowded scenes from tracklets, in: Computer Vision 
and Pattern Recognition (CVPR), IEEE Conference on, 2011, pp. 34413448.

[11] X. Zhao, G. Medioni, Robust unsupervised motion pattern inference from video and applications, in: International Conf. Computer Vision (ICCV), IEEE International Conference on, 2011, pp. 715-722.

[12] S. Ali, M. Shah, A lagrangian particle dynamics approach for crowd flow segmentation and stability analysis, in: Computer Vision and Pattern Recognition (CVPR), IEEE Conference on, 2007, pp. 1-6.

[13] D. Kuettel, M. D. Breitenstein, L. Van Gool, V. Ferrari, What's going on? discovering spatio-temporal dependencies in dynamic scenes, in: Computer Vision and Pattern Recognition (CVPR), IEEE Conference on, 2010, pp. 1951-1958.

[14] S. Zhang, H. Zhou, H. Yao, Y. Zhang, K. Wang, J. Zhang, Adaptive normalhedge for robust visual tracking, Signal Processing 110 (2015) $132-142$.

[15] S. Zhang, H. Zhou, F. Jiang, X. Li, Robust visual tracking using structurally random projection and weighted least squares, Circuits and Systems for Video Technology, IEEE Transactions on PP (2015) 1.

[16] S. Zhang, H. Yao, X. Sun, X. Lu, Sparse coding based visual tracking: review and experimental comparison, Pattern Recognition 46 (7) (2013) $1772-1788$.

[17] S. Zhang, H. Yao, H. Zhou, X. Sun, S. Liu, Robust visual tracking based on online learning sparse representation, Neurocomputing 100 (2013) $31-40$.

[18] W. Hu, D. Xie, Z. Fu, W. Zeng, S. Maybank, Semantic-based surveillance video retrieval, Image Processing 16 (4) (2007) 1168-1181.

[19] X. Wang, K. Tieu, E. Grimson, Learning semantic scene models by trajectory analysis, in: European Conf. Computer Vision (ECCV), Springer, 2006, pp. 110-123.

[20] F. Zhu, X. Wang, N. Yu, Crowd tracking with dynamic evolution of group structures, in: Computer Vision-ECCV 2014, Springer, 2014, pp. $139-154$. 
[21] B. Zhou, X. Tang, X. Wang, Measuring crowd collectiveness, in: Computer Vision and Pattern Recognition (CVPR), IEEE Conference on, 2013, pp. 3049-3056.

[22] X. Chen, Q. Ye, J. Zou, C. Li, Y. Cui, J. Jiao, Visual trajectory analysis via replicated softmax-based models, Signal, Image and Video Processing 8 (1) (2014) 183-190.

[23] A. Alahi, V. Ramanathan, L. Fei-Fei, Socially-aware large-scale crowd forecasting, in: Computer Vision and Pattern Recognition (CVPR), 2014 IEEE Conference on, IEEE, 2014, pp. 2211-2218.

[24] J. Zou, X. Chen, P. Wei, Z. Han, J. Jiao, A belief based correlated topic model for semantic region analysis in far-field video surveillance systems, in: Advances in Multimedia Information Processing (PCM), Springer, 2013, pp. 779-790.

[25] J. Zou, Q. Ye, Y. Cui, D. Doermann, J. Jiao, A belief based correlated topic model for trajectory clustering in crowded video scenes, in: International Conf. Pattern Recognition (ICPR), IEEE International Conference on, 2014, pp. 2543-2548.

[26] B. Zhou, X. Wang, X. Tang, Understanding collective crowd behaviors: Learning a mixture model of dynamic pedestrian-agents, in: Computer Vision and Pattern Recognition (CVPR), IEEE Conference on, 2012, pp. 2871-2878.

[27] M. Dixit, N. Rasiwasia, N. Vasconcelos, Class-specific simplex-latent dirichlet allocation for image classification, in: International Conf. Computer Vision (ICCV), IEEE Conference on, 2013, pp. 2672-2679.

[28] M. I. Jordan, Z. Ghahramani, T. S. Jaakkola, L. K. Saul, An introduction to variational methods for graphical models, Machine learning 37 (2) (1999) 183-233.

[29] M. Belkin, P. Niyogi, Laplacian eigenmaps for dimensionality reduction and data representation, Neural computation 15 (6) (2003) 1373-1396.

[30] S. Ali, Measuring flow complexity in videos, in: International Conf. Computer Vision (ICCV), IEEE International Conference on, 2013, pp. 1097-1104. 
[31] R. Mehran, B. E. Moore, M. Shah, A streakline representation of flow in crowded scenes, in: European Conf. Computer Vision (ECCV), Springer, 2010, pp. 439-452.

[32] Y. Zhang, L. Qin, R. Ji, H. Yao, Q. Huang, Social attribute-aware force model: Exploiting richness of interaction for abnormal crowd detection, Circuits and Systems for Video Technology, IEEE Transactions on PP (1) (2014) 1.

[33] X. Chong, W. Liu, P. Huang, N. I. Badler, Hierarchical crowd analysis and anomaly detection, Journal of Visual Languages \& Computing 25 (4) (2014) 376-393.

[34] S. Wu, H.-S. Wong, Z. Yu, A bayesian model for crowd escape behavior detection, Circuits and Systems for Video Technology, IEEE Transactions on 24 (1) (2014) 85-98.

[35] Y. Zhang, S. Zhang, Q. Huang, T. Serre, Learning sparse prototypes for crowd perception via ensemble coding mechanisms, in: Human Behavior Understanding, Springer, 2014, pp. 86-100.

[36] S. Yi, X. Wang, C. Lu, J. Jia, L0 regularized stationary time estimation for crowd group analysis, in: Computer Vision and Pattern Recognition (CVPR), 2014 IEEE Conference on, IEEE, 2014, pp. 2219-2226.

[37] A. Fagette, N. Courty, D. Racoceanu, J.-Y. Dufour, Unsupervised dense crowd detection by multiscale texture analysis, Pattern Recognition Letters 44 (2014) 126-133.

[38] B. Zhou, X. Tang, X. Wang, Coherent filtering: detecting coherent motions from crowd clutters, in: European Conf. Computer Vision (ECCV), Springer, 2012, pp. 857-871.

[39] Z. Shi, T. M. Hospedales, T. Xiang, Bayesian joint topic modelling for weakly supervised object localisation, in: International Conf. Computer Vision (ICCV), IEEE International Conference on, 2013, pp. 2984-2991.

[40] B. Zhao, L. Fei-Fei, E. P. Xing, Image segmentation with topic random field, in: European Conf. Computer Vision (ECCV), Springer, 2010, pp. $785-798$. 
[41] X. Wang, A. McCallum, Topics over time: a non-markov continuoustime model of topical trends, in: Proceedings of the 12th ACM SIGKD$\mathrm{D}$ international conference on Knowledge discovery and data mining, ACM, 2006, pp. 424-433.

[42] X. Wang, E. Grimson, Spatial latent dirichlet allocation, in: Advances in Neural Information Processing Systems, 2008, pp. 1577-1584.

[43] T. Hospedales, S. Gong, T. Xiang, Video behaviour mining using a dynamic topic model, International Journal of Computer Vision (IJCV) 98 (3) (2012) 303-323.

[44] J. Zhu, N. Chen, H. Perkins, B. Zhang, Gibbs max-margin topic models with data augmentation, The Journal of Machine Learning Research 15 (1) (2014) 1073-1110.

[45] Y. Wang, J. Zhu, Spectral methods for supervised topic models, in: Advances in Neural Information Processing Systems, 2014, pp. 15111519 .

[46] W. K. Hastings, Monte carlo sampling methods using markov chains and their applications, Biometrika 57 (1) (1970) 97-109.

[47] R. Liao, J. Zhu, Z. Qin, Nonparametric bayesian upstream supervised multi-modal topic models, in: Proceedings of the 7th ACM international conference on Web search and data mining, ACM, 2014, pp. 493-502.

[48] D. M. Blei, J. D. Lafferty, A correlated topic model of science, The Annals of Applied Statistics (2007) 17-35.

[49] M. Rodriguez, S. Ali, T. Kanade, Tracking in unstructured crowded scenes, in: International Conf. Computer Vision (ICCV), IEEE International Conference on, 2009, pp. 1389-1396.

[50] T. Carlo, T. Kanade, Detection and tracking of point features (1991).

[51] D. M. Blei, A. Y. Ng, M. I. Jordan, Latent dirichlet allocation, the Journal of machine Learning research 3 (2003) 993-1022.

[52] L. Fei-Fei, P. Perona, A bayesian hierarchical model for learning natural scene categories, in: Computer Vision and Pattern Recognition (CVPR), Vol. 2, IEEE Computer Society Conference on, 2005, pp. 524-531. 
[53] D. Cai, X. Wang, X. He, Probabilistic dyadic data analysis with local and global consistency, in: Proceedings of the 26th Annual International Conference on Machine Learning, ACM, 2009, pp. 105-112.

[54] F. Solera, S. Calderara, R. Cucchiara, Structured learning for detection of social groups in crowd, in: Advanced Video and Signal Based Surveillance (AVSS), IEEE International Conference on, 2013, pp. 7-12.

[55] C. C. Loy, T. M. Hospedales, T. Xiang, S. Gong, Stream-based joint exploration-exploitation active learning, in: Computer Vision and Pattern Recognition (CVPR), IEEE Conference on, 2012, pp. 1560-1567. 


\section{Appendix A.}

We detail the collective motion pattern inference in the proposed LC-LDA model.

Given the parameters $\alpha, \beta, \eta$, we write the joint probability with a mixture $\alpha$, a set of $N$ topics $z$, a set of $N$ points $x$, and the cluster $c$.

In Eq.1,

$$
\begin{gathered}
p(c \mid \eta)=\operatorname{Mult}(c \mid \eta) \\
p(\theta \mid c, \alpha)=\prod_{j=1}^{C} \operatorname{Dir}\left(\theta \mid \alpha_{j}\right)^{\delta(c, j)} \\
p\left(z_{j} \mid \theta_{j}\right)=\operatorname{Mult}\left(z_{j} \mid \theta_{j}\right), p(z \mid \theta) \propto \exp \left(\sum_{j} \log \theta_{j}+\sum_{j \in \delta(i)} D D\left(z_{\left.\left.j n 1, z_{i n 2}\right)\right)}\right.\right. \\
p\left(x_{j} \mid z_{j}, \beta\right)=\prod_{k=1}^{K} p\left(x_{j} \mid \beta_{k}\right)^{\delta\left(z_{j}^{k}, 1\right)}
\end{gathered}
$$

Given $x$, the cluster label $c^{\prime} s$ probability is:

$$
p(c \mid x, \theta, \beta, \eta) \propto p(x \mid c, \theta, \beta) \cdot p(c \mid \eta) \propto p(x \mid c, \theta, \beta) .
$$

For simplicity, the distribution of $p(c \mid \eta)$ is fixed where $p(c)=1 / C$. We omit the estimation of parameter $\eta$. $c$ is selected according to $\arg \max _{c} p(x \mid c, \theta, \beta)$. The goal is to maximize the $\log$ posterior probability $\log p(x \mid \alpha, \beta, \eta)$. Because we omit the estimation of $\eta$, the object function is changed to $\log p(x \mid \alpha, \beta, c)$ from $\log p(x \mid \alpha, \beta, \eta)$. We bound the $\log$ likelihood $\log p(x \mid \alpha, \beta, c)$ through Jensen's inequality as

$$
\begin{aligned}
\log p(x \mid \alpha, \beta, c) & =\log \int \sum_{z} p(\theta, z, x \mid \alpha, \beta, c) d \theta \\
& =\log \int \sum_{z} \frac{p(\theta, z, x \mid \alpha, \beta, c) q\left(\theta, z \mid \phi^{c}, \gamma^{c}\right)}{q\left(\theta, z \mid \phi^{c}, \gamma^{c}\right)} d \theta \\
& \geq \int \sum_{z} q\left(\theta, z \mid \phi^{c}, \gamma^{c}\right) \log p(\theta, z, x \mid \alpha, \beta, c) \\
& -\int \sum_{z} q\left(\theta, z \mid \phi^{c}, \gamma^{c}\right) \log q\left(\theta, z \mid \phi^{c}, \gamma^{c}\right) d \theta \\
& =\mathrm{E}_{q}[\log p(\theta, z, x \mid \alpha, \beta, c)]-E_{q}\left[\log q\left(\theta, z \mid \phi^{c}, \gamma^{c}\right)\right],
\end{aligned}
$$

where the term $q($.$) comes from the approximated distribution whose graphi-$ cal representation is shown in Figure 5(c). We use the term $L\left(\phi^{c}, \gamma^{c} ; \alpha, \beta\right)$ to denote the right-hand side of Eq.A.5. As shown in Eq.A.6, the deviation between $L\left(\phi^{c}, \gamma^{c} ; \alpha, \beta\right)$ and $\log p(x \mid \alpha, \beta, c)$ is $K L\left(q\left(\theta, z \mid \gamma^{c}, \phi^{c}\right)|| p(\theta, z \mid x, \alpha, \beta, c)\right)$ 
, where the term $K L($.$) denotes the KL divergence between the original dis-$ tribution and the approximated distribution. It can be concluded that if we iteratively optimize the term $L($.$) instead of \log p(x \mid \alpha, \beta, c)$, the term $K L($. gradually decreases. The smaller the term $K L($.$) is, the more closely the$ approximated distribution is approaching to the original distribution shown in Eq.3.

For further details, we factorize the term $L($.$) according to Figure 5(b),$ Figure 5(c) and Eq.A.6:

$$
\begin{aligned}
L\left(\phi^{c}, \gamma^{c} ; \alpha, \beta\right) & =E_{q}[\log p(\theta \mid \alpha, c)]+E_{q}[\log p(z \mid \theta)]+E_{q}[\log p(x \mid z, \beta)] \\
& -E_{q}\left[\log q\left(\theta \mid \gamma^{c}\right)\right]-E_{q}\left[\log q\left(\mathrm{z} \mid \phi^{c}\right)\right] .
\end{aligned}
$$

In LC-LDA, we implement the locally consistent constraints to generate a new object function in Eq.4. In Eq.5, $R$ is a term where a symmetrical KL divergence measurement is implemented between tracklet $p$ and $q$ to measure the similarity with their probabilities. $W_{p, q}$ is a neighbor graph through the KNN method, which can model the local geometric structure on the manifold. In Eq.6, $N_{K}\left(d_{q}\right)$ denotes the set of $K$ nearest neighbors of $d_{p}$ , where neighbor sets are computed according to the Euclidean distance.

According to the definition of KL divergence in Eq.7, term $R$ is detailed in Eq.8.

For simplicity, we replace $1-\frac{\phi_{q, n, k}^{c}}{\phi_{p, n, k}^{c}}$ with $\log \left(\frac{\phi_{p, n, k}^{c}}{\phi_{q, n, k}^{c}}\right)$. Considering the terms relating to motion pattern inference only, we only list the derivation of $\beta, \phi$. Please refer to [51] for the computation of other terms. We firstly maximize Eq.8 through the derivation of $L^{\prime}$ with respect to $\phi$, which is the probability that a word is generated by a latent topic. Naturally, the constrained condition $\sum_{k=1}^{K} \phi_{k, n}=1$ must be held. Therefore, we reform $L^{\prime}$ by isolating the terms that contain $\phi$ and adding the Lagrange multipliers as

$$
\begin{gathered}
L_{\left[\phi_{p, n, k}^{c}\right]}^{\prime}=\phi_{p, n, k}^{c}\left(\Psi\left(\gamma_{k}\right)-\Psi\left(\sum_{k=1}^{K} \gamma_{k}\right)\right)+\phi_{p, n, k}^{c} \log \beta_{k, v}-\phi_{p, n, k}^{c} \log \phi_{p, n, k}^{c}+ \\
\rho_{p, n}\left(\sum_{k=1}^{K} \phi_{p, n, k}^{c}-1\right)-\frac{\lambda}{2} \sum_{s}^{M}\left(\sum_{k}^{K} \phi_{q, n, k}^{c} \log \frac{\phi_{q, n, k}^{c}}{\phi_{p, n, k}^{c}}+\sum_{k}^{K} \phi_{p, n, k}^{c} \log \frac{\phi_{p, n, k}^{c}}{\phi_{q, n, k}^{c}}\right) W_{p, q}
\end{gathered}
$$

where $\rho_{p, n}$ is the Lagrangian parameter and $\lambda$ is the regularization factor of locally consistent constraints. Taking derivatives of $L_{\left[\phi_{p, n, k}^{c}\right]}$ with respect to 
$\phi_{p, n, k}^{c}$, we obtain the following:

$$
\begin{aligned}
\frac{\partial L_{\left[\phi_{p, n, k}^{\prime}\right]}^{c}}{\partial \phi_{p, n, k}^{c}} & =\Psi\left(\gamma_{k}^{c}\right)-\Psi\left(\sum_{k=1}^{K} \gamma_{k}^{c}\right)+\log \beta_{k, v}-\log \phi_{p, n, k}^{c}-1 \\
& +\rho_{i n}-\frac{\lambda}{2} \sum_{s}^{M} \sum_{k}^{K}\left(1-\frac{\phi_{q, n, k}^{c}}{\phi_{p, n, k}^{c}}+\log \frac{\phi_{p, n, k}^{c}}{\phi_{q, n, k}^{c}}\right) W_{p, q}
\end{aligned}
$$

where $\Psi$ is the digamma function, the first derivative of the log gamma function. Setting this derivative to zero yields the maximising value of the variational parameter $\phi_{p, n, k}^{c}$ shown in Eq.11.

The parameter $\rho_{p, n}$ cannot be omitted because of the locally consistent constraints. $\rho_{p, n}$ is computed as

$$
\begin{aligned}
\rho_{p, n}= & 1-\left(1+\lambda \sum_{s=1}^{M} W_{p, q}\right) * \\
& \log \left\{\sum_{k=1}^{K} \exp \left(\frac{\Psi\left(\gamma_{k}^{c}\right)-\Psi\left(\sum_{k=1}^{K} \gamma_{k}^{c}\right)+\log \beta_{k, v}+\lambda \sum_{s=1}^{M} \sum_{k}^{K} \log \phi_{q, n, k}^{c} W_{p, q}}{1+\lambda \sum_{s=1}^{M} W_{p, q}}\right)\right\} .
\end{aligned}
$$

$\beta$ does not change significantly, compared to the term in [51] as

$$
\log \beta_{n, k} \propto \sum_{p}\left(\log \phi_{p, n, k}^{c}+\log n_{p}\right) .
$$

